\title{
Non-Biologic Disease-Modifying Antirheumatic Drug
}

National Cancer Institute

\section{Source}

National Cancer Institute. Non-Biologic Disease-Modifying Antirheumatic Drug. NCI

Thesaurus. Code C121333.

A drug used to alter the progression of autoimmune disease that is not a biologic. 